\title{
Os sistemas de informação de saúde no Brasil e o direito à intimidade
}

\author{
The health information systems in Brazil and the right to privacy
}

\section{Sandra Mara Campos Alves}

Advogada, Especialista em Direito Sanitário e Mestre em Política Social. Pesquisadora Colaboradora do Programa de Direito Sanitário da Fundação Oswaldo Cruz. Brasília, Brasil.

Resumo: A informação em saúde ocupa papel estratégico na sociedade contemporânea, com o surgimento de inúmeros sistemas de informação que transformam essas informações em insumos estratégicos para a gestão, o planejamento e a avaliação da situação de saúde da população. Por outro lado, o desenvolvimento e a difusão de novas tecnologias da informação e comunicação a nível global - caracterizados por usar predominantemente tecnologias da informação para a troca de informação em formato digital, de fácil manipulação, reprodução e transmissão - gera importantes problemas de proteção da intimidade e privacidade do cidadão, nelas incluídas as informações de saúde. A publicação, no Brasil, da Lei $n^{\circ} 12.527$, de 18 de novembro de 2011, abre uma nova discussão sobre os limites e contornos do direito de acesso à informação, impondo-se uma análise detalhada sobre o tema. No âmbito do Direito Sanitário, a discussão é importante uma vez que a informação em saúde é insumo para a produção da legislação sanitária e o acesso à informação sanitária também é relevante para o fortalecimento de ações de controle social, um dos pilares do sistema de saúde no Brasil.

Palavras chave: Lei de Acesso à Informação; Direito Sanitário; sistemas de informação em saúde.

Resumen: La información de salud ocupa un papel estratégico en la sociedad contemporánea, con la aparición de numerosos sistemas de información que transforman esas informaciones en insumos estratégicos para la gestión, la planificación y la evaluación del estado de salud de la población. Por otra parte, el desarrollo y la difusión de las nuevas tecnologías de información u comunicación a nivel mundial - caracterizados por el mayor uso de la tecnología de información para el intercambio de información en formato digital, fácil de usar, reproducir y transmitir crea problemas importantes para la protección de la intimidad y privacidad de los ciudadanos, incluidos en ellas las informaciones de salud. La publicación, en Brasil, de la Ley $\mathrm{n}^{\circ} 12.527$, del 18 de noviembre de 2011, abre un nuevo debate sobre los límites y contornos del derecho de acceso a la información, imponiendo un análisis detallado sobre el tema. En el ámbito del Derecho Sanitario, esa discusión es importante porque la información de salud es insumo para la producción de la legislación sanitaria y el acceso a la información de salud también es relevante para el fortalecimiento de las acciones de control social, uno de los pilares del sistema público de salud en Brasil. 
Palabras clave: Acceso a la Información; Derecho Sanitario; sistemas de información de salud.

\begin{abstract}
The health information occupies a strategic role in contemporary society, with the emergence of numerous health information systems that transform that information into strategic inputs for the management, planning and evaluation of health status of the population. Moreover, the development and diffusion of new technologies of information and communication on a global level - characterized by predominantly use information technology to exchange information in digital format, easy to use, reproduction and transmission - creates major problems for the protection of intimacy and privacy of the citizen, therein including health information. The publication, in Brazil, of Law no. 12.527, of November 18, 2011, opens a new discussion on the limits and contours of the right of access to information, imposing a detailed analysis on the subject. Under the Health Law, this discussion is important because health information is input for the production of sanitary legislation and the access to health information is relevant to strengthening actions of social control, one of the pillars of the public health system in Brazil.
\end{abstract}

Keywords: Access to Information Act; Health Law; health information systems.

\title{
Introdução
}

O desenvolvimento e a difusão de novas tecnologias de informação e comunicação (TICs) estão trazendo um dinamismo cada vez maior na geração e difusão de informações, e no caso da saúde, esse processo não é distinto. Nesse contexto, inúmeros sistemas de informações em saúde são hoje operados pelos Estados, produzindo uma gama de informações fundamentais ao processo de tomada de decisão do gestor público, e na implementação de políticas públicas de saúde.

No plano internacional, observa-se o reconhecimento do direito humano ao acesso às informações constantes em órgãos e entidades públicas, sem que esse seja absoluto, pois esbarra em limitações justificadas por questões de segurança da sociedade e do Estado, e também pelo aspecto do direito à privacidade.

No Brasil, com a publicação da Lei $n^{\circ} 12.527$ de 18 de novembro de 2011, que cuidou de regular o acesso à informação constitucionalmente previsto, abre-se uma nova discussão sobre os limites e contornos do direito de acesso à informação.

Nesse sentido, o presente artigo aborda o tema das informações pessoais contidas nos sistemas públicos de informação em saúde, diante do binômio direito à informação e o direito à privacidade.

Sem a pretensão de esgotar a discussão, pretende-se com o presente texto trazer a discussão para o âmbito do direito sanitário, uma vez que a informação em saúde é insumo para a produção da legislação sanitária. Ademais, o acesso à 
informação sanitária também é relevante para o fortalecimento de ações de controle social, um dos pilares do sistema de saúde no Brasil.

\section{A informação como produto econômico}

Desde o final do século XX, a nível global, a sociedade experimenta o surgimento de uma nova economia, conhecida como a economia da informação ou do imaterial, que se fundamenta em um novo paradigma tecnológico.

Castells (2005, p. 119) se refere a essa nova economia como um "processo de descontinuidade histórica", onde a força da economia que se centrava anteriormente em uma organização produtiva agrária e industrial, é substituída por um novo produto, que é a própria informação.

Essa alteração foi possível a partir do surgimento do computador na década de 50, e posteriormente da internet, que possibilitou novas formas de coleta, armazenamento, distribuição e aplicação desse produto, surgindo então a informação digital (Castells, 2005).

Essa "nova Revolução Industrial" também cria uma nova sociedade, a sociedade da informação, onde a informação se torna um bem de consumo semelhante a qualquer outro bem material, tornando-se um fator importante na organização econômica e social dos estados. "A informação é, hoje, fator determinante da história da humanidade. [...] Como decorrência dessa valorização da informação, num processo irreversível, o desenrolar das atividades humanas, em todas as instâncias, depende cada vez mais da gestão técnica e científica" (Targino, 2009, p. 53).

A sociedade da informação se caracteriza por usar, predominantemente tecnologias da informação e conhecimento (TIC) para troca de informação em formato digital, de fácil manipulação, reprodução e transmissão. Esse avanço tecnológico e a disseminação do uso das novas tecnologias acarretam mudanças não apenas no comportamento social e cultural dos indivíduos, mas na própria reorganização da atividade produtiva dos estados.

As redes sociais são o exemplo atual mais significativo de alteração de padrões - social, cultural e econômico - advindos do uso dessas novas tecnologias de informação e conhecimento.

No campo da saúde, o impacto da utilização das tecnologias da informação e conhecimento é bastante conhecido, em especial no desenvolvimento de novas 
tecnologias de diagnóstico e tratamento de inúmeras doenças. Áreas como a bioestatística e a epidemiologia também têm se beneficiado com esses avanços, e a partir de banco de dados digitais cada vez maiores e mais robustos, são capazes de produzir análises cada vez mais detalhadas, e que são utilizadas na gestão e estruturação da atenção à saúde (Carvalho, 2009; Targino, 2009).

Ademais, a informação em saúde auxilia o processo de tomada de decisão em sede de políticas públicas, pois permite identificar problemas individuais e coletivos, que interferem diretamente na qualidade de vida da população. Informações sobre morbidade, taxa de mortalidade infantil, características demográficas, etc., são relevantes não apenas para a solução de questões relacionadas ao processo saúdedoença, mas também temas de natureza administrativa, de gestão pública.

Nesse sentido é o entendimento de Dallari (2007, p. 638) sobre os bancos de dados epidemiológicos, e a importância para o entendimento do processo saúdedoença: "[...] o conhecimento científico sobre as doenças e o estado de saúde das populações está, cada vez mais, baseado no acúmulo de informações específicas sobre o tema. Assim, quanto maior o número de variáveis que se puder conhecer e controlar a respeito de cada evento do processo saúde/doença, maior a possibilidade de compreender esse processo, suas causas e suas condições. A existência de bancos de dados que permitam a identificação do indivíduo, de seu ambiente social, físico e, muitas vezes, econômico, representa um instrumento insubstituível para o trabalho do epidemiologista. $E$ também isso é do interesse da sociedade como um todo".

Inegável, portanto que a informação em saúde ocupa papel estratégico na sociedade contemporânea, o que permitiu o surgimento de inúmeros sistemas de informação em saúde que transformam esses dados em insumos estratégicos para a gestão, planejamento e avaliação da situação de saúde da população, em grande escala.

Esses sistemas de informações em saúde guardam, em sua base de dados, não apenas dados epidemiológicos, mas também dados clínicos, coletados diretamente do cidadão, quando do atendimento individual em serviços de saúde. São, portanto, banco de dados que envolvem informações públicas e privadas.

Nesse sentido, a reflexão sob o acesso às informações contidas nessa base de dados deve ser feita levando-se em consideração o binômio direito à informação e o direito à privacidade. 
O direito sanitário se ocupa desse campo de análise porque o acesso à informação sanitária é relevante para a produção de dados epidemiológicos que vão influir diretamente na elaboração e análise de políticas públicas de saúde, sem desconsiderar, entretanto, o aspecto privado dessa informação.

\section{0 direito humano à informação}

No plano internacional, a informação como direito humano tem seus primeiros delineamentos na Declaração Universal dos Direitos Humanos de 1948, que reconhece no direito à liberdade de opinião e expressão, o direito de sem interferências, procurar, receber e transmitir informações e ideias, por qualquer meio, e independente de fronteiras (art. 19).

Em 1966, o direito à informação é novamente referido em documentos internacionais, desta vez no Pacto Internacional dos Direitos Civis e Políticos (art. 19), que em linhas gerais, confirma e reforça o que já havia sido reconhecido na Declaração de Direitos Humanos de 1948.

Inúmeros outros documentos internacionais reforçam e reconhecem a importância do direito à informação como a Declaração sobre o Direito e a Responsabilidade dos Indivíduos, Grupos ou Órgãos da Sociedade de Promover e Proteger os Direitos Humanos e Liberdades Fundamentais Universalmente Reconhecidos, produzida pela ONU em 1998 e a Convenção das Nações Unidas contra a Corrupção em 2003, vislumbrando consequências positivas do seu exercício para o fortalecimento da democracia, a emancipação do indivíduo e a prevenção e combate a corrupção, ou seja, o exercício do direito à informação está diretamente imbricado com o fortalecimento da própria sociedade e da cultura de transparência da Administração Pública.

A transparência é condição essencial aos Estados Democráticos de Direito, como é o caso do Brasil, e se concretiza por meio do acesso à informação pelo cidadão. Nesse sentido, tão importante quanto a produção da informação, em especial no aspecto de educação e formação de uma consciência crítica da sociedade, é o acesso a essa informação (Medeiros et alli, 2014).

O princípio da transparência na Administração Pública, segundo Martins (2011), comporta duas dimensões: ativa e passiva. A transparência ativa deve ser compreendida como a divulgação espontânea das informações que estão na guarda 
ou são produzidas pelo Estado; ao passo que na transparência passiva, o Poder Público apresenta as informações quando instado pelo indivíduo.

E para que haja o pleno exercício do direito à informação, não basta apenas que os órgãos públicos produzam informações, ou que respondam aos pedidos que são formulados. É necessária uma ação proativa desses órgãos na publicação e divulgação voluntária dessas informações.

Eventuais limitações ao direito à informação devem ser excepcionais, e justificadas por situações onde o dano real e objetivo que possa ser causado pela divulgação de dados supere o interesse público.

\section{0 direito à informação no contexto brasileiro: as informações de saúde}

No Brasil, a saúde é reconhecida no plano constitucional como um direito social, que deve ser garantido pelo Estado mediante políticas sociais e econômicas (Brasil, 1988).

Para a garantia do direito social à saúde é estratégica a utilização das informações de saúde contidas nos bancos de dados. O uso dessas informações nas políticas públicas de saúde pode gerar redução de riscos ao adoecimento, melhoria na qualidade de vida da população, o que legitima a coleta e uso de informações pessoais para o estabelecimento de informações sanitárias relevantes, como estabelecimento de hábitos que podem causar agravo ou redução de riscos à saúde.

Segundo Panitz (2012), desde a segunda metade dos anos de 1970, surgem, no Brasil, os primeiros sistemas de informação públicos em saúde. E atualmente, com o crescimento das tecnologias de informação e comunicação, existe cerca de 54 (cinquenta e quatro) sistemas de informação principais ligados ao Ministério da Saúde, que coletam e difundem dados para as mais diversas áreas da saúde (Panitz, 2012).

A Constituição Federal brasileira garante o direito de acesso à informação (art. $\left.5^{\circ}, \mathrm{XIV}\right)$, e mais especificamente, o direito de receber informações de órgãos públicos, seja de interesse particular, ou de interesse geral ou coletivo (art. $5^{\circ}, \mathrm{XXXIII)}$ (Brasil, 1998).

O texto constitucional também apresenta os fundamentos do direito à intimidade e privacidade, ao dispor que "são invioláveis a intimidade, a vida privada, a honra e a imagem das pessoas [...]" (art. 50, X CF/88). Esses direitos garantem ao indivíduo repelir a interferência de terceiros em sua esfera pessoal, inclusive no 
tocante à divulgação de informações ao seu respeito. Nesse sentido podem ser limitadores do exercício do direito de acesso à informação.

Em 2011 foi publicada a Lei $n^{\circ} 12.527$ com o objetivo de estabelecer diretrizes e regulamentar o direito de acesso à informação produzida pelo Estado, ou sob sua guarda.

A "lei de acesso à informação" (LAI), como vem sendo conhecida, se estrutura a partir de cinco diretrizes:

Art. $3^{\circ}$. Os procedimentos previstos nesta Lei destinam-se a assegurar o direito fundamental de acesso à informação e devem ser executados em conformidade com os princípios básicos da administração pública e com as seguintes diretrizes:

I - observância da publicidade como preceito geral e do sigilo como exceção;

II - divulgação de informações de interesse público, independentemente de solicitações;

III - utilização de meios de comunicação viabilizados pela tecnologia da informação;

IV - fomento ao desenvolvimento da cultura de transparência na administração pública;

V - desenvolvimento do controle social da administração pública. (Brasil, 2011)

Observa-se que as diretrizes apontam para uma primazia da publicidade das informações referentes às atividades estatais, em detrimento do sigilo, reforçando assim o princípio da transparência na Administração Pública, além de possibilitar o fortalecimento de ações de controle social.

A Lei $n^{\circ} 12.527 / 2011$ trás em seu texto o reconhecimento do princípio da transparência ativa ao dispor que é dever dos órgãos e entidades públicas a promoção da divulgação, independente de requerimento, de informações de interesse coletivo ou geral, que forem produzidas ou custodiadas por eles. Ainda em conformidade com o texto legal, uma das formas de divulgação dessas informações é por meio dos sites oficiais (internet).

Outro ponto importante a ser destacado é que a LAl estabelece de um rol mínimo de informações que devem estar disponíveis ao público, quando do exercício da transparência ativa pelos órgãos e entidades públicas.

Essas informações vão desde temas relacionados ao registro de despesas, procedimentos de licitação, transferência de recursos financeiros, até dados gerais das unidades como endereço, telefone e horário de atendimento ao público. 
As restrições impostas pela Lei no 12.527/2011 ao acesso à informação vão desde questões de segurança nacional, às limitações em virtude do caráter personalíssimo da informação.

Segundo a LAI, são consideradas imprescindíveis às questões de segurança da sociedade e do Estado, e por isso passíveis de classificação quanto ao grau de sigilo (reservado, secreto e ultrassecreto), informações que possam por em risco: a) a defesa e a soberania nacionais ou a integridade do território nacional; b) a condução de negociações ou as relações internacionais do País, ou as que tenham sido fornecidas em caráter sigiloso por outros Estados e organismos internacionais; c) a vida, a segurança ou a saúde da população; d) à estabilidade financeira, econômica ou monetária do País; e) planos ou operações estratégicos das Forças Armadas; f) segurança de instituições ou de altas autoridades nacionais ou estrangeiras e seus familiares e; g) atividades de inteligência, bem como de investigação ou fiscalização em andamento, relacionadas com a prevenção ou repressão de infrações.

No caso específico de divulgação ou acesso por terceiros às informações pessoais, relativas à intimidade, à vida privada, à honra e à imagem, a LAl dispõe que ficam condicionados a expressa autorização do titular da informação, ou mediante previsão legal.

Contudo, essa restrição no direito de acesso às informações de caráter pessoal também comporta exceção. O consentimento expresso do titular das informações pessoais é dispensado em situações onde o legislador entendeu que o interesse público deve prevalecer.

Assim, é dispensada a autorização expressa do titular da informação, nos casos onde as informações de caráter pessoal sejam necessárias: a) à prevenção e diagnóstico médico, desde que o titular da informação esteja física ou legalmente incapaz, e que o uso da informação seja exclusivamente para o tratamento médico; b) à realização de estatísticas e pesquisas científicas de interesse público ou geral, sendo nesse caso, vedada a identificação pessoal; c) ao cumprimento de ordem judicial; d) à defesa de direitos humanos; e, e) proteção do interesse público e geral.

Os inúmeros sistemas de informação públicos em saúde são compostos por dados pessoais, coletados nos momentos de atendimentos individuais realizados em serviços públicos de saúde, sejam eles de atenção básica, média ou alta complexidade. São, portanto dados pessoais do cidadão que serão utilizados para o levantamento estatístico e epidemiológico de determinadas situações de saúde, 
É inegável a importância estratégica dessas informações no que concerne a reflexão das práticas de saúde, da situação de saúde, e da própria política de saúde adotada, pois serão utilizadas como substrato para proposição ou alteração da política pública de saúde, colaborando diretamente com o processo de tomada de decisão do gestor da saúde, estando plenamente atendido o requisito do interesse público e geral preponderante.

Ademais, a LAl autoriza o uso dessas informações, desde que suprimida a identificação da pessoa a quem as informações se referirem, o que já é observado pelos sistemas públicos de informações em saúde utilizados no Brasil.

Nesse sentido, há que se estimular o uso das tecnologias de informação e comunicação na produção de sistemas de informação em saúde cada vez mais intercambiáveis, de modo a produzir informação qualificada que possa ser utilizada pelo tomador de decisão para a concretização do direito à saúde.

\section{Considerações finais}

O direito de acesso à informação, em especial a informação em saúde, deve ser objeto de reflexão e discussão a partir de novos paradigmas, condizentes com a sociedade da informação que atualmente experimentamos, e alinhados ao arcabouço jurídico internacional que reconhece o direito humano à informação como imprescindível ao fortalecimento da cidadania e da democracia.

A informação em saúde é fator determinante no empoderamento não apenas da sociedade, para o aprimoramento do controle social, mas também para o tomador de decisão, de modo a qualificar suas ações. Deste modo, os sistemas de informação em saúde podem ser percebidos como ferramentas que auxiliam nesse processo.

Nesse sentido, e em desconsiderar os aspectos da intimidade, vida privada, honra e imagem, as informações de caráter pessoal podem e devem ser utilizadas para compor os sistemas de informação em saúde. É por meio dessas informações que se desenha o cenário real da situação de saúde de uma determinada coletividade, de modo a poder nela intervir, seja para sua manutenção ou melhoria, contribuindo para a realização do direito à saúde.

\section{Referências}

BRASIL. Constituição Federal de 1988. <Disponível na internet na URL: http://www.planalto.gov.br/ccivil_03/constituicao/constituicaocompilado.htm> (Consultado em 5 abr. 2013). 
BRASIL. Lei $n^{\circ} 12.527$, de 18 de novembro de 2011. <Disponível na internet na URL: http://www.planalto.gov.br/ccivil_03/_Ato2011-2014/2011/Lei/L12527.htm> (Consultado em 3 abr. 2013).

CARVALHO, André Luis Bonifácio. Informação em saúde como ferramenta estratégica para a qualificação da gestão e o fortalecimento do controle social no SUS. Tempus Actas de Saúde Coletiva, 3(3):16-30, jun./set. 2009.

CASTELLS, Manuel. Sociedade em rede. São Paulo : Paz e Terra, 2005. ISBN 9788577530366

DALLARI, Sueli Gandolfi. A justiça, o direito e os bancos de dados epidemiológicos. Ciência \& Saúde Coletiva, 12(3):633-641, 2007. ISSN 1413-8123

MARTINS, Paula Lígia. Acesso à informação: Um direito fundamental e instrumental. Acervo, Rio de Janeiro, 24(1):233-244, jan./jun. 2011. ISSN 2237-8723

MEDEIROS, Simone Assis; MAGALHÃES, Roberto e PEREIRA, José Roberto. Lei de acesso à informação: em busca da transparência e do combate à corrupção. Informação \& Informação, Londrina, 19(1):55-75, jan./abr. 2014. ISSN 1981-8920

PANITZ, Leandro Manassi. As informações da atenção à saúde no Brasil e a lei de acesso à informações: a informação que temos e a informação que queremos. Monografia (Curso de Especialização em Direito Sanitário). Fundação Oswaldo Cruz, Brasília, 2012.

TARGINO, Maria das Graças. Informação em Saúde: potencialidades e limitações. Informação \& Informação, Londrina, 14(1):52-81, jun./jul. 2009. ISSN 1981-8920

VENTURA, Mirian. Lei de acesso à informação, privacidade e a pesquisa em saúde. Cadernos de Saúde Pública, Rio de Janeiro, 29(4):636-638, abr. 2013. ISSN 16784464. 